\title{
Editorial: Miniaturized Bioenergy and Energy Harvesting Systems
}

\author{
Hao Ren ${ }^{1 *}$, Junrui Liang ${ }^{1 *}$, Jicheng Feng ${ }^{2 *}$ and Xiaosheng Zhang ${ }^{3 *}$ \\ ${ }^{1}$ School of Information Science and Technology, ShanghaiTech University, Shanghai, China, ${ }^{2}$ School of Physical Science and \\ Technology, ShanghaiTech University, Shanghai, China, ${ }^{3}$ School of Electronic Science and Engineering, University of Electronic \\ Science and Technology of China, Chengdu, China
}

Keywords: microsystems, energy harvesting, piezoelectric energy harvester, self-powered system, ambient energy, bioenergy

Editorial on the Research Topic

\section{Miniaturized Bioenergy and Energy Harvesting Systems}

Miniaturized energy harvesting systems, such as miniaturized microbial fuel cells, biological fuel cells, triboelectric energy harvesters, piezoelectric energy harvesters, represent an emerging area of research and have found a great potential for developing self-powered wireless sensor networks (WSN), Internet of Things (IoT), and portable electronics applications. Nowadays, power supply remains one of the major critical issues for the development of WSN and IoT. Semiconductor chips in the systems enjoy the scaling effect from miniaturization. The scaling effect means that when the

\section{OPEN ACCESS}

Edited and reviewed by: Ajay Giri Prakash Kottapalli, University of Groningen, Netherlands

*Correspondence: Hao Ren renhao@shanghaitech.edu.cn Junrui Liang liangjr@shanghaitech.edu.cn Jicheng Feng

fengjch@shanghaitech.edu.cn Xiaosheng Zhang

zhangxs@uestc.edu.cn

Specialty section: This article was submitted to Micro- and Nanoelectromechanical Systems,

a section of the journal Frontiers in Mechanical Engineering

Received: 14 July 2021 Accepted: 28 September 2021 Published: 22 October 2021

Citation:

Ren $\mathrm{H}$, Liang J, Feng $J$ and Zhang $X$ (2021) Editorial: Miniaturized Bioenergy and Energy Harvesting Systems.

Front. Mech. Eng 7:741190. doi: 10.3389/fmech.2021.741190 size of devices is small, they have a higher surface area to volume ratio, smaller size, less expense, and faster frequency response. As a result, a variety of miniaturized sensors, actuators, and microsystems have taken advantage of the scaling effect, such as microprocessors, accelerometers, gyroscopes, micromirrors, actuators, biosensors, and resonators, etc. (Yazdi et al., 1998; Chae et al., 2005; Hu et al., 2010; Ren et al., 2011; Ren et al., 2013; Wang et al., 2014; Yeap et al., 2019; Fei and Ren, 2021). However, traditional power supplies, such as lithium-ion batteries, suffer from lower capacity due to miniaturization and often require frequent replacement after deployment. As a result, it is critical to develop miniaturized energy harvesting systems, which are self-sustainable, for these emerging applications.

Microbial fuel cells are bionic-based electrochemical fuel cells that directly convert the chemical energy stored in organic compounds from biomass into electrical energy (Logan and Rabaey, 2012; Ren et al., 2015; Ren et al., 2016a; Ren et al., 2016b). This is accomplished through the catalytic reaction of specific microorganisms called exoelectrogens or Anode-Respiring Bacteria (ARB) (Torres et al., 2008). Microbial fuel cell represents a carbon-neutral and renewable energy converter. Miniaturized MFCs have smaller chamber volumes compared with macro or mesoscale MFCs, and they are generally fabricated by microfabrication techniques. The surface area to volume ratio of miniaturized MFCs is high, resulting in a higher current and power density (Ren et al., 2012). Although the current and power densities significantly boosted in the past 2 decades, however, the current and power densities of miniaturized microbial fuel cells are still lower than conventional energy conversion and storage devices, such as lithium-ion batteries. The approaches which have the potential to further improve the current and power density of miniaturized microbial fuel cells are discussed in this topic by (Ren).

Biological fuel cells are fuel cells that convert chemical energy stored in organic compounds into electricity with the catalytic activity of biological enzymes or living entities. Compared with conventional energy conversion and storage devices, such as the lithium-ion battery, biological fuel cells can utilize the organic compounds in the environment and living entities, and it also represents a carbon-neutral and renewable energy converter (Li et al., 2020a). Miniaturized biological 
fuel cells utilize microfabrication and microfluidics techniques to reduce their size and they have the potential to be implemented into power sources for implantable medical devices. In this research topic, Maza et al. present a low-cost glucose $/ \mathrm{O}_{2}$ Y-shaped microfluidic biofuel cell that was developed using a printed circuit board (Mashayekhi Mazar et al., 2021). They implemented a double-side tape based on the pressuresensitive adhesive to fabricate the microfluidic channel for the microfluidic biofuel cell. The electrode was coated with a nanocomposite that consisted of reduced graphene oxide gold nanoparticles (AuNPs). Aspergillus niger glucose oxidase enzyme and Mytheliophthora thermophile laccase were used to modify the anodic and cathodic electrodes. A maximum power density of $36 \mu \mathrm{W} / \mathrm{cm}^{2}$ and an open-circuit voltage of $0.5 \mathrm{~V}$ are reported at a flow rate of $50 \mu \mathrm{L} / \mathrm{min}$.

Triboelectric energy harvesters, also named triboelectric nanogenerator (TENG), implement triboelectricity, which is static-electricity charges generated by contact and motion between surfaces. When two materials are brought into contact and separated, the electric charge separation and induction process occur, and electricity is generated. Since its invention in 2012 (Fan et al., 2012), triboelectric energy harvesters have found many applications in self-powered systems, such as wearable sensors (Wen et al., 2019), trajectory-tracking microsystems (Ba et al., 2021), magnetic sensors (Yang et al., 2012), chemical sensors (Huang et al., 2021), etc. In this research topic, Zhao and Zhu discuss the application of triboelectric nanogenerator in smart home and clothing applications (Zhao and Zhu). For smart home applications, triboelectric energy harvesters can be implemented for switch sensors for smart home lighting fixtures, pressure sensors for home floors, power supply for home security systems, etc. For clothing applications, triboelectric energy harvesters can be implemented for sensing the frequency and acceleration when integrated into shoe sole, absorbing static electricity on clothes and reducing the static electricity on clothes, and sensing gestures when integrated into smart gloves, etc.

Piezoelectric energy harvesters utilize the piezoelectric effect to convert mechanical energy into electrical energy. The

\section{REFERENCES}

Ba, Y.-Y., Bao, J.-F., Wang, Z.-Y., Deng, H.-T., Wen, D.-L., Zhang, X.-R., et al. (2021). Self-powered Trajectory-Tracking Microsystem Based on ElectrodeMiniaturized Triboelectric Nanogenerator. Nano Energy 82, 105730. doi:10.1016/j.nanoen.2020.105730

Fan, F.-R., Tian, Z.-Q., and Lin Wang, Z. (2012). Flexible Triboelectric Generator. Nano Energy 1, 328-334. doi:10.1016/ j.nanoen.2012.01.004

Fei, S., and Ren, H. (2021). Temperature Characteristics of a Contour Mode MEMS AlN Piezoelectric Ring Resonator on SOI Substrate. Micromachines 12, 143. doi: $10.3390 / \mathrm{mi} 12020143$

Hu, F., Yao, J., Qiu, C., and Ren, H. (2010). A MEMS Micromirror Driven by Electrostatic Force. J. Electrostatics 68, 237-242. doi:10.1016/j.elstat.2010.01.005

Huang, C., Chen, G., Nashalian, A., and Chen, J. (2021). Advances in Self-Powered Chemical Sensing via a Triboelectric Nanogenerator. Nanoscale 13, 2065-2081. doi:10.1039/d0nr07770d piezoelectric effect arises when a strain is produced on piezoelectric materials. Positive and negative charges accumulate on the two opposite surfaces of the piezoelectric materials, respectively. If we connect a load between the two surfaces with opposite charges, current flows. Thus mechanical strain energy is converted into electrical energy. Nowadays, piezoelectric energy harvesters have been widely implemented for self-powered systems and IoT applications, such as vibration- or motion-powered sensing and transmitting systems (Li et al., 2020b), self-powered motion detection systems (Li et al., 2021), and self-powered wearable upper limb (Liu et al., 2021). In this research topic, Guo et al. presented a theoretical and experimental study of the vibration dynamics of a 3D-printed sandwich beam with an hourglass lattice truss core. It has a provided potential solution to explore the advantages of using sandwich beams for piezoelectric energy harvesting (Guo et al.).

In addition to the miniaturized energy harvesters, power management systems are also critical as the output power from the energy harvesters cannot be directly used to drive the loads. Power management electronics are necessary for energy harvesting systems. The power management circuits aim to convert the output of the energy harvesters to voltage levels which can be directly fed to load, such as wireless sensors or IoT devices. A variety of power management systems for microbial fuel cells, biological fuel cells, triboelectric energy harvesters, and piezoelectric energy harvesters have been reported (Liang and Liao, 2011; Zhang et al., 2014; Xu et al., 2017; Liang et al., 2018; Liang et al., 2019). Due to the advantage of self-powering, carbon-neutral, and renewable characteristics, the miniaturized bioenergy and energy harvesting systems integrated with power management circuits may provide solutions to powering billions of WSN or IoT systems in the future.

\section{AUTHOR CONTRIBUTIONS}

HR wrote the original editorial, JL, JF, and $\mathrm{XZ}$ revised the editorial.

Chae, J., Kulah, H., and Najafi, K. (2005). A Monolithic Three-axis Micro-g Micromachined Silicon Capacitive Accelerometer. J. Microelectromech. Syst. 14, 235-242. doi:10.1109/jmems.2004.839347

Li, X., Lv, P., Yao, Y., Feng, Q., Mensah, A., Li, D., et al. (2020). A Novel SingleEnzymatic Biofuel Cell Based on Highly Flexible Conductive Bacterial Cellulose Electrode Utilizing Pollutants as Fuel. Chem. Eng. J. 379, 122316. doi:10.1016/ j.cej.2019.122316

Li, X., Tang, H., Hu, G., Zhao, B., and Liang, J. (2021). ViPSN-pluck: A TransientMotion-Powered Motion Detector. IEEE Internet Things J., 1. doi:10.1109/ jiot.2021.3098238

Li, X., Teng, L., Tang, H., Chen, J., Wang, H., Liu, Y., et al. (2020). ViPSN: A Vibration-Powered IoT Platform. IEEE Internet of Things Journal.

Liang, J., and Liao, W.-H. (2011). Improved Design and Analysis of Self-Powered Synchronized Switch Interface Circuit for Piezoelectric Energy Harvesting Systems. IEEE Trans. Ind. Electronics 59, 1950-1960.

Liang, J., Zhao, Y., and Zhao, K. (2018). Synchronized Triple Bias-Flip Interface Circuit for Piezoelectric Energy Harvesting Enhancement. IEEE Trans. Power Electronics 34, 275-286. 
Liang, X., Jiang, T., Liu, G., Xiao, T., Xu, L., Li, W., et al. (2019). Triboelectric Nanogenerator Networks Integrated with Power Management Module for Water Wave Energy Harvesting. Adv. Funct. Mater. 29, 1807241. doi:10.1002/adfm.201807241

Liu, Y., Khanbareh, H., Halim, M. A., Feeney, A., Zhang, X., Heidari, H., et al. (2021). Piezoelectric Energy Harvesting for Self-powered Wearable Upper Limb Applications. Nano Select.

Logan, B. E., and Rabaey, K. (2012). Conversion of Wastes into Bioelectricity and Chemicals by Using Microbial Electrochemical Technologies. Science 337, 686-690. doi:10.1126/science.1217412

Ren, H., Lee, H.-S., and Chae, J. (2012). Miniaturizing Microbial Fuel Cells for Potential Portable Power Sources: Promises and Challenges. Microfluid Nanofluid 13, 353-381. doi:10.1007/s10404-012-0986-7

Ren, H., Pyo, S., Lee, J.-I., Park, T.-J., Gittleson, F. S., Leung, F. C. C., et al. (2015). A High Power Density Miniaturized Microbial Fuel Cell Having Carbon Nanotube Anodes. J. Power Sourc. 273, 823-830. doi:10.1016/j.jpowsour.2014.09.165

Ren, H., Rangaswami, S., Lee, H.-S., and Chae, J. (2016). Enhanced Current and Power Density of Micro-scale Microbial Fuel Cells with Ultramicroelectrode Anodes. J. Micromech. Microeng. 26, 095016. doi:10.1088/0960-1317/26/9/095016

Ren, H., Tao, F., Wang, W., and Yao, J. (2011). An Out-Of-Plane Electrostatic Actuator Based on the Lever Principle. J. Micromech. Microeng. 21, 045019. doi:10.1088/0960-1317/21/4/045019

Ren, H., Tian, H., Gardner, C. L., Ren, T.-L., and Chae, J. (2016). A Miniaturized Microbial Fuel Cell with Three-Dimensional Graphene Macroporous Scaffold Anode Demonstrating a Record Power Density of over 10000 W M-3. Nanoscale 8, 3539-3547. doi:10.1039/c5nr07267k

Ren, H., Wang, W., Tao, F., and Yao, J. (2013). A Bi-directional Out-Of-Plane Actuator by Electrostatic Force. Micromachines 4, 431-443. doi:10.3390/ mi4040431

Torres, C. I., Kato Marcus, A., and Rittmann, B. E. (2008). Proton Transport inside the Biofilm Limits Electrical Current Generation by Anode-Respiring Bacteria. Biotechnol. Bioeng. 100, 872-881. doi:10.1002/bit.21821

Wang, R., Wang, W., Ren, H., and Chae, J. (2014). Detection of Copper Ions in Drinking Water Using the Competitive Adsorption of Proteins. Biosens. Bioelectron. 57, 179-185. doi:10.1016/j.bios.2014.01.056

Wen, D.-L., Liu, X., Deng, H.-T., Sun, D.-H., Qian, H.-Y., Brugger, J., et al. (2019). Printed Silk-Fibroin-Based Triboelectric Nanogenerators for
Multi-Functional Wearable Sensing. Nano Energy 66, 104123. doi:10.1016/j.nanoen.2019.104123

Xu, Z., Liu, Y., Williams, I., Li, Y., Qian, F., Wang, L., et al. (2017). Flat EnzymeBased Lactate Biofuel Cell Integrated with Power Management System: Towards Long Term In Situ Power Supply for Wearable Sensors. Appl. Energ. 194, 71-80. doi:10.1016/j.apenergy.2017.01.104

Yang, Y., Lin, L., Zhang, Y., Jing, Q., Hou, T.-C., and Wang, Z. L. (2012). Selfpowered Magnetic Sensor Based on a Triboelectric Nanogenerator. ACS Nano 6, 10378-10383. doi:10.1021/nn304374m

Yazdi, N., Ayazi, F., and Najafi, K. (1998). Micromachined Inertial Sensors. Proc. IEEE 86, 1640-1659. doi:10.1109/5.704269

Yeap, G., Lin, S., Chen, Y., Shang, H., Wang, P., Lin, H., et al. (2019). IEEE, 36.7. 1-36.7. 4.5nm CMOS Production Technology Platform Featuring Full-Fledged EUV, and High Mobility Channel FinFETs with Densest 0.021 Mm 2 SRAM Cells for Mobile SoC and High Performance Computing Applications2019 IEEE International Electron Devices Meeting (IEDM).

Zhang, X., Ren, H., Pyo, S., Lee, J.-I., Kim, J., and Chae, J. (2014). A High-Efficiency DC-DC Boost Converter for a Miniaturized Microbial Fuel Cell. IEEE Trans. Power Electronics 30, 2041-2049.

Conflict of Interest: The authors declare that the research was conducted in the absence of any commercial or financial relationships that could be construed as a potential conflict of interest.

Publisher's Note: All claims expressed in this article are solely those of the authors and do not necessarily represent those of their affiliated organizations, or those of the publisher, the editors and the reviewers. Any product that may be evaluated in this article, or claim that may be made by its manufacturer, is not guaranteed or endorsed by the publisher.

Copyright (c) 2021 Ren, Liang, Feng and Zhang. This is an open-access article distributed under the terms of the Creative Commons Attribution License (CC BY). The use, distribution or reproduction in other forums is permitted, provided the original author(s) and the copyright owner(s) are credited and that the original publication in this journal is cited, in accordance with accepted academic practice. No use, distribution or reproduction is permitted which does not comply with these terms. 